\title{
NON-CONSTANT POSITIVE STEADY STATES FOR A STRONGLY COUPLED NONLINEAR REACTION-DIFFUSION SYSTEM ARISING IN POPULATION DYNAMICS
}

\author{
ZIJUAN WEN AND YUAN QI
}

\begin{abstract}
We consider a strongly coupled reactiondiffusion system describing three interacting species in a simple food chain structure. Based on the Leray-Schauder degree theory, the existence of non-constant positive steady states is investigated. The results indicate that, when the intrinsic growth rate of the middle species is small, crossdiffusions of the predators versus the preys are helpful to create global coexistence (stationary patterns).
\end{abstract}

1. Introduction. Let $\Omega$ be a fixed bounded domain in $\mathbb{R}^{N}$ with smooth boundary $\partial \Omega$. Denote $u_{1}, u_{2}$ and $u_{3}$ as the population densities of three interacting species. In this paper, we investigate an ecosystem arising in population dynamics in which the third species preys on the second one and simultaneously the second species preys on the first one. Namely, the following three-species food chain system will be considered:

$$
\left\{\begin{array}{l}
u_{1 t}-\Delta\left[\left(d_{1}+\alpha_{12} u_{2}\right) u_{1}\right]=f_{1} \quad \text { in } \Omega \times(0, \infty), \\
u_{2 t}-\Delta\left[\left(d_{2}+\alpha_{21} u_{1}+\alpha_{23} u_{3}\right) u_{2}\right]=f_{2} \quad \text { in } \Omega \times(0, \infty), \\
u_{3 t}-\Delta\left[\left(d_{3}+\alpha_{32} u_{2}\right) u_{3}\right]=f_{3} \quad \text { in } \Omega \times(0, \infty), \\
\frac{\partial u_{1}}{\partial \nu}=\frac{\partial u_{2}}{\partial \nu}=\frac{\partial u_{3}}{\partial \nu}=0 \quad \text { on } \partial \Omega \times(0, \infty) \\
u_{i}(x, 0)=u_{i 0}(x) \text { in } \Omega \text { for } i=1,2,3
\end{array}\right.
$$

2010 AMS Mathematics subject classification. Primary 35J57, Secondary 35B35, 92D25.

Keywords and phrases. Diffusion, cross-diffusion, food chain, non-constant positive steady states, stationary pattern formation.

The first author is supported by the National Natural Science Foundation of China (No. 11201204).

Received by the editors on March 3, 2011, and in revised form on August 12, 2013. 
with

$$
\begin{aligned}
& f_{1}=f_{1}\left(u_{1}, u_{2}\right)=u_{1}\left(a_{1}-b_{11} u_{1}-b_{12} u_{2}\right), \\
& f_{2}=f_{2}\left(u_{1}, u_{2}, u_{3}\right)=u_{2}\left(a_{2}+b_{21} u_{1}-b_{22} u_{2}-b_{23} u_{3}\right), \\
& f_{3}=f_{3}\left(u_{2}, u_{3}\right)=u_{3}\left(a_{3}+b_{32} u_{2}-b_{33} u_{3}\right)
\end{aligned}
$$

where $\nu$ is the unit outward normal to $\partial \Omega, d_{i}, a_{i}, b_{i i}, i=1,2,3, \alpha_{12}, \alpha_{21}$, $\alpha_{23}, \alpha_{32}, b_{12}, b_{21}, b_{23}$ and $b_{32}$ are all positive constants, $u_{i 0}, i=1,2,3$ are nonnegative functions which are not identically zero. $a_{i}$ denotes the intrinsic growth rate of the $i$ th species and $b_{i i}$ accounts for intraspecific competitions, while $b_{12}, b_{21}, b_{23}$ and $b_{32}$ are the coefficients for inter-specific interactions. $d_{i}$ is the diffusion rate of the $i$ th species, and $\alpha_{i j}(i \neq j)$ is cross-diffusion pressure of the $i$ th species due to the presence of the $j$ th species. Diffusion is population pressure due to the mutual interference between the individuals, describing the migration of species to avoid crowds. Cross-diffusion expresses the population flux of one species due to the presence of the other species. For more details on the biological backgrounds of this model, see also [5, 24].

Problem (1.1) describes a strongly coupled parabolic system in a food web with three species chasing in succession. The system is self contained with no population flux across the boundary, the population of which is not homogeneously distributed due to the consideration of diffusions. Through global bifurcation techniques, the authors of [20] discussed the coexistence of weakly coupled steady state problem of (1.1) with Dirichlet boundary conditions. Moreover, the global stability of the Neumann problem was investigated. Based on this, further results about the coexistence of steady state problem of equations in (1.1) with cross-diffusions and homogeneous Dirichlet boundary conditions were obtained in [15]. As for the time-dependent solution of (1.1), the authors of [6] investigated the global existence of the nonnegative weak solution and the non-existence of non-constant positive steady state.

The strongly coupled population model with Lotka-Volterra type reaction terms was proposed by Shigesada, Kawasaki and Teramoto in 1979 and is known as the SKT model [29]. Since then, the twospecies SKT competing system and its overall behavior continue to be of great interest in literature to both mathematical analysis and real-life modelling $[\mathbf{1}, \mathbf{8}, \mathbf{1 9}, \mathbf{2 2}, \mathbf{2 3}, \mathbf{2 8}, \mathbf{3 1}]$. But more and more attention has 
been recently focused on three or multi-species systems and the SKT model in any space dimension due to their more complicated patterns. Meanwhile, the SKT models with other types of reaction terms are also proposed and investigated $[2,3,7,12,16,17,18,21,25$, $\mathbf{2 6}, \mathbf{2 7}, \mathbf{3 2}]$. The results obtained mainly concentrate on two aspects, namely, the stability analysis of constant positive steady states and the existence of non-constant positive steady states (stationary patterns) $[2,3,12,15,16,17,18,21,22,23,25,26,27,28,31,32]$, and the global existence of non-negative time-dependent solutions $[\mathbf{1}, \mathbf{6}, \mathbf{8}, \mathbf{1 9}]$.

What is of great interest in the competition and predator-prey systems is whether the various species can coexist. In the case that the species are homogeneously distributed, coexistence indicates that the mathematical model ultimately reaches a constant positive equilibrium, whereas in the case that the scatter of species is not homogeneous, the coexistence state is denoted by the existence of a non-constant positive steady state, which is called stationary pattern formation.

Starting with Turing's seminal paper [30] in 1952, diffusion and cross-diffusion have been observed as causes of the spontaneous emergence of ordered structures, namely, stationary patterns. Since the 1980's, many authors have investigated the existence of stationary patterns for various reaction-diffusion systems arising in population dynamics and have obtained plenty of research results $[2,3,4,11,12$, $13,14,15,16,17,18,20,21,22,23,25,26,27,28,32]$. As for the ecological systems with cross-diffusions and Lotka-Volterra type reaction terms, a pioneering work [22] studied the effects of diffusion, self-diffusion and cross-diffusion of the two-species SKT competition model. The results show that there is no non-constant steady state if diffusion or self-diffusion is strong, or if cross-diffusion is weak, while non-constant positive steady states do exist if cross-diffusions are suitably strong. Moreover, the authors of [25] firstly began to investigate the three species predator-prey model with cross-diffusion and found that stationary patterns do not emerge from the diffusion of individual species but only appear with the introduction of cross-diffusion. They, together with the other references mentioned above, all confirmed the role of cross-diffusion in helping to create stationary patterns.

Typically, there are three approaches for investigating the coexistence states of elliptic systems. One is the global bifurcation technique, for instance, see [4] for the two-species competition model, 
$[\mathbf{1 2}, \mathbf{1 6}, \mathbf{1 7}, \mathbf{2 7}]$ for the two-species prey-predator model and $[\mathbf{1 1}, \mathbf{2 0}]$ for the three-species prey-predator systems. A variation of the bifurcation technique is the Leray-Schauder degree theoretic approach. In this direction, see $[\mathbf{2 1}, \mathbf{2 2}, \mathbf{2 3}]$ for two and three species competition systems, $[\mathbf{3}, \mathbf{2 5}, \mathbf{2 7}, \mathbf{3 2}]$ for two and three species prey-predator model and [2] for the competition-competition-mutualist model. The other two approaches are singular perturbation and the upper and lower solutions method, see $[\mathbf{1 3}, \mathbf{1 4}, \mathbf{2 6}, \mathbf{2 8}]$, respectively.

In this paper, we intend to establish the existence of non-constant positive steady states of system (1.1) through using Leray-Schauder degree theory. The paper will be organized as follows. In Section 2, we discuss the stability of positive equilibria of ODEs and PDEs, respectively. Moreover, the non-existence of the non-constant positive steady state for (1.1) with vanished cross-diffusions will be obtained. In Section 3, we obtain a priori upper and lower bounds for the positive steady states of (1.1) in order to calculate the topology degree. In Section 4, we establish the global existence of the non-constant positive steady state of (1.1) for suitable values of cross-diffusion coefficients $\alpha_{21}$ and $\alpha_{32}$. Our results show that global coexistence is likely to occur for the small intrinsic growth rate of the middle species and large crossdiffusions of the predators versus the preys, which confirms a pushing effect of cross-diffusion on stationary pattern formation.

2. Stability of positive equilibria of ODEs and PDEs. Consider the following ODE system:

$$
\left\{\begin{array}{l}
\frac{d u_{1}}{d t}=u_{1}\left(a_{1}-b_{11} u_{1}-b_{12} u_{2}\right), \\
\frac{d u_{2}}{d t}=u_{2}\left(a_{2}+b_{21} u_{1}-b_{22} u_{2}-b_{23} u_{3}\right), \\
\frac{d u_{3}}{d t}=u_{3}\left(a_{3}+b_{32} u_{2}-b_{33} u_{3}\right), \\
u_{i}(0)=u_{0 i} \text { in } \Omega \text { for } i=1,2,3 .
\end{array}\right.
$$

It is known that the solution $\mathbf{u}=\left(u_{1}, u_{2}, u_{3}\right)^{\mathrm{T}}$ of system (2.1) is nonnegative and bounded for all $t \geq 0$ when the initial value condition $\left(u_{01}, u_{02}, u_{03}\right)^{\mathrm{T}} \geq \mathbf{0}$ holds. Moreover, if

$$
\left\{\begin{array}{l}
a_{1} b_{22} b_{33}+a_{1} b_{23} b_{32}+a_{3} b_{12} b_{23}>a_{2} b_{12} b_{33}, \\
a_{1} b_{21} b_{33}+a_{2} b_{11} b_{33}>a_{3} b_{11} b_{23},
\end{array}\right.
$$


then the positive equilibrium of (2.1) is uniquely given by

$$
\begin{aligned}
\bar{u}_{1} & =\frac{a_{1} b_{22} b_{33}+a_{1} b_{23} b_{32}+a_{3} b_{12} b_{23}-a_{2} b_{12} b_{33}}{b_{11} b_{22} b_{33}+b_{11} b_{23} b_{32}+b_{12} b_{21} b_{33}}, \\
\bar{u}_{2} & =\frac{a_{1} b_{21} b_{33}+a_{2} b_{11} b_{33}-a_{3} b_{11} b_{23}}{b_{11} b_{22} b_{33}+b_{11} b_{23} b_{32}+b_{12} b_{21} b_{33}}, \\
\bar{u}_{3} & =\frac{a_{1} b_{21} b_{32}+a_{2} b_{11} b_{32}+a_{3} b_{11} b_{22}+a_{3} b_{12} b_{21}}{b_{11} b_{22} b_{33}+b_{11} b_{23} b_{32}+b_{12} b_{21} b_{33}} .
\end{aligned}
$$

Denote $\overline{\mathbf{u}}=\left(\bar{u}_{1}, \bar{u}_{2}, \bar{u}_{3}\right)^{\mathrm{T}}$.

Proposition 2.1. Assume the parameters in (2.1) satisfy (2.2). The equilibrium $\overline{\mathbf{u}}$ of (2.1) is globally asymptotically stable.

Proof. Define

$$
\begin{aligned}
E(t)= & E(\overline{\mathbf{u}})(t)=\left(u_{1}-\bar{u}_{1}-\bar{u}_{1} \ln \frac{u_{1}}{\bar{u}_{1}}\right) \\
& +\rho_{1}\left(u_{2}-\bar{u}_{2}-\bar{u}_{2} \ln \frac{u_{2}}{\bar{u}_{2}}\right) \\
& +\rho_{2}\left(u_{3}-\bar{u}_{3}-\bar{u}_{3} \ln \frac{u_{3}}{\bar{u}_{3}}\right),
\end{aligned}
$$

where $\rho_{1}=\left(b_{12} b_{21}+b_{11} b_{22}\right) b_{21}^{-2}, \quad \rho_{2}=\left(b_{12} b_{21}+b_{11} b_{22}\right)\left(b_{23} b_{32}+\right.$ $\left.b_{22} b_{33}\right) b_{21}^{-2} b_{32}^{-2}$. It is obvious that $E(\overline{\mathbf{u}}) \geq 0$, and $E(\overline{\mathbf{u}})=0$ if and only if $\mathbf{u}=\overline{\mathbf{u}}$. Referring to (2.1), we compute

$$
\begin{aligned}
\frac{d E}{d t}= & \frac{u_{1}-\bar{u}_{1}}{u_{1}} u_{1}^{\prime}+\rho_{1} \frac{u_{2}-\bar{u}_{2}}{u_{2}} u_{2}^{\prime}+\rho_{2} \frac{u_{3}-\bar{u}_{3}}{u_{3}} u_{3}^{\prime} \\
= & \left(u_{1}-\bar{u}_{1}\right)\left(a_{1}-b_{11} u_{1}-b_{12} u_{2}\right) \\
& +\rho_{1}\left(u_{2}-\bar{u}_{2}\right)\left(a_{2}+b_{21} u_{1}-b_{22} u_{2}-b_{23} u_{3}\right) \\
& +\rho_{2}\left(u_{3}-\bar{u}_{3}\right)\left(a_{3}+b_{32} u_{2}-b_{33} u_{3}\right) \\
= & -\left[b_{11}\left(u_{1}-\bar{u}_{1}\right)^{2}+\left(b_{12}-b_{21} \rho_{1}\right)\left(u_{1}-\bar{u}_{1}\right)\left(u_{2}-\bar{u}_{2}\right)\right. \\
& +\rho_{1} b_{22}\left(u_{2}-\bar{u}_{2}\right)^{2}+\left(b_{23} \rho_{1}-b_{32} \rho_{2}\right)\left(u_{2}-\bar{u}_{2}\right)\left(u_{3}-\bar{u}_{3}\right) \\
& \left.+\rho_{2} b_{33}\left(u_{3}-\bar{u}_{3}\right)^{2}\right] \\
\leq & -\delta\left[\left(u_{1}-\bar{u}_{1}\right)^{2}+\left(u_{2}-\bar{u}_{2}\right)^{2}+\left(u_{3}-\bar{u}_{3}\right)^{2}\right]<0 .
\end{aligned}
$$


The above inequalities are obtained since the positive definiteness of quadratic form $b_{11}\left(u_{1}-\bar{u}_{1}\right)^{2}+\left(b_{12}-b_{21} \rho_{1}\right)\left(u_{1}-\bar{u}_{1}\right)\left(u_{2}-\bar{u}_{2}\right)+$ $\rho_{1} b_{22}\left(u_{2}-\bar{u}_{2}\right)^{2}+\left(b_{23} \rho_{1}-b_{32} \rho_{2}\right)\left(u_{2}-\bar{u}_{2}\right)\left(u_{3}-\bar{u}_{3}\right)+\rho_{2} b_{33}\left(u_{3}-\bar{u}_{3}\right)^{2}$. By the Lyapunov-LaSalle invariance principle in [10], $\overline{\mathbf{u}}$ is globally asymptotically stable.

On the other hand, we investigate the local stability of constant positive steady state for the reaction-diffusion system (1.1). For simplicity, we denote

$$
\begin{aligned}
\mathbf{\Phi}(\mathbf{u})= & \left(\phi_{1}(\mathbf{u}), \phi_{2}(\mathbf{u}), \phi_{3}(\mathbf{u})\right)^{\mathrm{T}} \\
= & \left(\left(d_{1}+\alpha_{12} u_{2}\right) u_{1},\left(d_{2}+\alpha_{21} u_{1}+\alpha_{23} u_{3}\right) u_{2},\left(d_{3}+\alpha_{32} u_{2}\right) u_{3}\right)^{\mathrm{T}}, \\
\mathbf{F}(\mathbf{u})= & \left(F_{1}(\mathbf{u}), F_{2}(\mathbf{u}), F_{3}(\mathbf{u})\right)^{\mathrm{T}} \\
= & \left(u_{1}\left(a_{1}-b_{11} u_{1}-b_{12} u_{2}\right), u_{2}\left(a_{2}+b_{21} u_{1}-b_{22} u_{2}-b_{23} u_{3}\right),\right. \\
& \left.\quad u_{3}\left(a_{3}+b_{32} u_{2}-b_{33} u_{3}\right)\right)^{\mathrm{T}} .
\end{aligned}
$$

Then the problem (1.1) reduces to

$$
\left\{\begin{array}{l}
\frac{\partial \mathbf{u}}{\partial t}-\Delta \mathbf{\Phi}(\mathbf{u})=\mathbf{F}(\mathbf{u}) \text { in } \Omega \times(0, \infty), \\
\frac{\partial \mathbf{u}}{\partial \nu}=0 \text { on } \partial \Omega \times(0, \infty), \\
\mathbf{u}(x, 0)=\left(u_{10}(x), u_{20}(x), u_{30}(x)\right)^{\mathrm{T}} \text { in } \Omega,
\end{array}\right.
$$

and the linearization of problem (2.3) at the positive equilibrium $\overline{\mathbf{u}}$ is

$$
\left\{\begin{array}{l}
\frac{\partial \mathbf{u}}{\partial t}-\boldsymbol{\Phi}_{\mathbf{u}}(\overline{\mathbf{u}}) \Delta \mathbf{u}=\mathbf{F}_{\mathbf{u}}(\overline{\mathbf{u}}) \mathbf{u} \text { in } \Omega \times(0, \infty) \\
\frac{\partial \mathbf{u}}{\partial \nu}=0 \text { on } \partial \Omega \times(0, \infty) \\
\mathbf{u}(x, 0)=\left(u_{10}(x), u_{20}(x), u_{30}(x)\right)^{\mathrm{T}} \text { in } \Omega
\end{array}\right.
$$

where

$$
\boldsymbol{\Phi}_{\mathbf{u}}(\overline{\mathbf{u}})=\left(\begin{array}{ccc}
d_{1}+\alpha_{12} \bar{u}_{2} & \alpha_{12} \bar{u}_{1} & 0 \\
\alpha_{21} \bar{u}_{2} & d_{2}+\alpha_{21} \bar{u}_{1}+\alpha_{23} \bar{u}_{3} & \alpha_{23} \bar{u}_{2} \\
0 & \alpha_{32} \bar{u}_{3} & d_{3}+\alpha_{32} \bar{u}_{2}
\end{array}\right)
$$

and

$$
\mathbf{F}_{\mathbf{u}}(\overline{\mathbf{u}})=\left(\begin{array}{ccc}
-b_{11} \bar{u}_{1} & -b_{12} \bar{u}_{1} & 0 \\
b_{21} \bar{u}_{2} & -b_{22} \bar{u}_{2} & -b_{23} \bar{u}_{2} \\
0 & b_{32} \bar{u}_{3} & -b_{33} \bar{u}_{3}
\end{array}\right)
$$


Let $\left\{\lambda_{i}, \varphi_{i}\right\}_{i=1}^{\infty}$ be a set of eigenpairs for $-\Delta$ in $\Omega$ with no flux boundary condition, where $0=\lambda_{1}<\lambda_{2} \leq \lambda_{3} \leq \cdots$, and let $E\left(\lambda_{i}\right)$ be the eigenspace corresponding to $\lambda_{i}$ in $H^{1}(\bar{\Omega}), \varphi_{i j}, j=1, \ldots, \operatorname{dim} E\left(\lambda_{i}\right)$, be an orthonormal basis of $E\left(\lambda_{i}\right)$. Let $\mathbf{X}=\left\{\mathbf{u} \in\left[H^{1}(\bar{\Omega})\right]^{3} \mid(\partial \mathbf{u} / \partial \nu)=\right.$ $\mathbf{0}$ on $\partial \Omega\}$ and $\mathbf{X}_{i j}=\left\{\mathbf{c} \varphi_{i j} \mid \mathbf{c} \in \mathbb{R}^{3}\right\}$. Then we can do the following decomposition:

$$
\mathbf{X}=\bigoplus_{i=1}^{\infty} \mathbf{X}_{i}, \text { where } \mathbf{X}_{i}=\bigoplus_{j=1}^{\operatorname{dim} E\left(\lambda_{i}\right)} \mathbf{X}_{i j}
$$

For each $i \geq 1, \mathbf{X}_{i}$ is invariant under the operator $\mathfrak{L}=\boldsymbol{\Phi}_{\mathbf{u}}(\overline{\mathbf{u}}) \Delta+$ $\mathbf{F}_{\mathbf{u}}(\overline{\mathbf{u}})$. Then problem (2.4) has a non-trivial solution of the form $\mathbf{u}=\mathbf{c} \phi \exp \{\mu t\}$, where $\mathbf{c} \in \mathbb{R}^{3}$ is a constant vector, if and only if $(\mu, \mathbf{c})$ is an eigenpair for the matrix $-\lambda_{i} \boldsymbol{\Phi}_{\mathbf{u}}(\overline{\mathbf{u}})+\mathbf{F}_{\mathbf{u}}(\overline{\mathbf{u}})$.

The characteristic equation of matrix $-\lambda_{i} \boldsymbol{\Phi}_{\mathbf{u}}(\overline{\mathbf{u}})+\mathbf{F}_{\mathbf{u}}(\overline{\mathbf{u}})$ is given by

$$
p_{i}(\mu)=\mu^{3}+B_{2, i} \mu^{2}+B_{1, i} \mu+B_{0, i}=0,
$$

where

$$
\begin{aligned}
B_{2, i}= & \left(d_{1}+\alpha_{12} \bar{u}_{2}+d_{2}+\alpha_{21} \bar{u}_{1}+\alpha_{23} \bar{u}_{3}+d_{3}+\alpha_{32} \bar{u}_{2}\right) \lambda_{i}+b_{11} \bar{u}_{1} \\
+ & b_{22} \bar{u}_{2}+b_{33} \bar{u}_{3}>0 \\
B_{1, i}= & {\left[\left(d_{2}+\alpha_{21} \bar{u}_{1}\right)\left(d_{3}+\alpha_{32} \bar{u}_{2}\right)+d_{3} \alpha_{23} \bar{u}_{3}\right.} \\
& +d_{1}\left(d_{2}+\alpha_{21} \bar{u}_{1}+\alpha_{23} \bar{u}_{3}+d_{3}+\alpha_{32} \bar{u}_{2}\right)+\alpha_{12} \bar{u}_{2}\left(d_{2}+\alpha_{23} \bar{u}_{3}\right. \\
& \left.\left.+d_{3}+\alpha_{32} \bar{u}_{2}\right)\right] \lambda_{i}^{2}+\left[b_{11} \bar{u}_{1}\left(d_{2}+\alpha_{21} \bar{u}_{1}+\alpha_{23} \bar{u}_{3}+d_{3}+\alpha_{32} \bar{u}_{2}\right)\right. \\
& +b_{22} \bar{u}_{2}\left(d_{3}\right. \\
+ & \left.\alpha_{32} \bar{u}_{2}\right)+b_{33} \bar{u}_{3}\left(d_{2}+\alpha_{21} \bar{u}_{1}+\alpha_{23} \bar{u}_{3}\right) \\
& +\left(b_{22} \bar{u}_{2}+b_{33} \bar{u}_{3}\right)\left(d_{1}+\alpha_{12} \bar{u}_{2}\right) \\
& \left.+\left(\alpha_{23} b_{32}-\alpha_{32} b_{23}\right) \bar{u}_{2} \bar{u}_{3}+\left(\alpha_{12} b_{21}-\alpha_{21} b_{12}\right) \bar{u}_{1} \bar{u}_{2}\right] \lambda_{i} \\
& +\left(b_{11} b_{22}+b_{12} b_{21}\right) \bar{u}_{1} \bar{u}_{2}+\left(b_{22} b_{33}+b_{23} b_{32}\right) \bar{u}_{2} \bar{u}_{3}+b_{11} b_{33} \bar{u}_{1} \bar{u}_{3}, \\
B_{0, i}= & {\left[\left(d_{1} d_{2}+d_{1} \alpha_{21} \bar{u}_{1}+d_{2} \alpha_{12} \bar{u}_{2}\right)\left(d_{3}+\alpha_{32} \bar{u}_{2}\right)\right.} \\
& \left.+d_{3} \alpha_{23} \bar{u}_{3}\left(d_{1}+\alpha_{12} \bar{u}_{2}\right)\right] \lambda_{i}^{3}+\left[b_{33} \bar{u}_{3}\left(d_{1}+\alpha_{12} \bar{u}_{2}\right)\left(d_{2} \alpha_{23} \bar{u}_{3}\right)\right. \\
& +d_{1} \alpha_{21} b_{33} \bar{u}_{1} \bar{u}_{3}+b_{22} \bar{u}_{2}\left(d_{1}+\alpha_{12} \bar{u}_{2}\right)\left(d_{3}+\alpha_{32} \bar{u}_{2}\right) \\
& +\left(\alpha_{23} b_{32}-\alpha_{32} b_{23}\right) \bar{u}_{2} \bar{u}_{3}\left(d_{1}\right. \\
& \left.+\alpha_{12} \bar{u}_{2}\right)+\left(\alpha_{12} b_{21}-\alpha_{21} b_{12}\right) \bar{u}_{1} \bar{u}_{2}\left(d_{3}+\alpha_{32} \bar{u}_{2}\right)+d_{3} b_{11} \bar{u}_{1}\left(d_{2}\right.
\end{aligned}
$$




$$
\begin{aligned}
& \left.\left.+\alpha_{21} \bar{u}_{1}+\alpha_{23} \bar{u}_{3}\right)+b_{11} \alpha_{32} \bar{u}_{1} \bar{u}_{2}\left(d_{2}+\alpha_{21} \bar{u}_{1}\right)\right] \lambda_{i}^{2} \\
& +\left[\left(b_{11} b_{22}+b_{12} b_{21}\right)\left(d_{3}+\alpha_{32} \bar{u}_{2}\right) \bar{u}_{1} \bar{u}_{2}\right. \\
& +\left(b_{22} b_{33}+b_{23} b_{32}\right)\left(d_{1}+\alpha_{12} \bar{u}_{2}\right) \bar{u}_{2} \bar{u}_{3} \\
& +b_{11} b_{33}\left(d_{2}+\alpha_{21} \bar{u}_{1}+\alpha_{23} \bar{u}_{3}\right) \bar{u}_{1} \bar{u}_{3} \\
& \left.+\left(\left(\alpha_{12} b_{21}-\alpha_{21} b_{12}\right) b_{33}+\left(\alpha_{23} b_{32}-\alpha_{32} b_{23}\right) b_{11}\right) \bar{u}_{1} \bar{u}_{2} \bar{u}_{3}\right] \lambda_{i} \\
& +\left(b_{11} b_{22} b_{33}+b_{12} b_{21} b_{33}+b_{11} b_{23} b_{32}\right) \bar{u}_{1} \bar{u}_{2} \bar{u}_{3} .
\end{aligned}
$$

Obviously, if

$$
\alpha_{12} b_{21} \geq \alpha_{21} b_{12}, \quad \alpha_{23} b_{32} \geq \alpha_{32} b_{23},
$$

then $B_{1, i}>0, B_{0, i}>0$. Moreover, an elementary calculation shows that $B_{2, i} B_{1, i}-B_{0, i}>0$ for all $i \geq 1$. It follows from the Routh-Hurwitz criterion that the three roots $\mu_{1, i}, \mu_{2, i}$ and $\mu_{3, i}$ of $p_{i}(\mu)=0$ all have negative real parts for all $i \geq 1$.

In order to obtain the local stability of $\overline{\mathbf{u}}$, we need to prove that there exists a positive constant $\delta$ such that

$$
\operatorname{Re}\left\{\mu_{1, i}\right\}, \operatorname{Re}\left\{\mu_{2, i}\right\}, \operatorname{Re}\left\{\mu_{3, i}\right\} \leq-\delta \quad \text { for all } i \geq 1 \text {. }
$$

Let $\mu=\lambda_{i} \zeta$. Then

$$
p_{i}(\mu)=\lambda_{i}^{3} \zeta^{3}+B_{2, i} \lambda_{i}^{2} \zeta^{2}+B_{1, i} \lambda_{i} \zeta+B_{0, i} \triangleq \widetilde{p}_{i}(\zeta) .
$$

Notice that $\lambda_{i} \rightarrow \infty$ as $i \rightarrow 0$. Through a simple calculation, we can obtain

$$
\begin{aligned}
\lim _{i \rightarrow 0} \frac{\widetilde{p}_{i}(\mu)}{\lambda_{i}^{3}}= & \zeta^{3}+\left(d_{1}+\alpha_{12} \bar{u}_{2}+d_{2}+\alpha_{21} \bar{u}_{1}+\alpha_{23} \bar{u}_{2}\right) \zeta^{2} \\
& +\left[\left(d_{2}+\alpha_{21} \bar{u}_{1}\right)\left(d_{3}+\alpha_{32} \bar{u}_{2}\right)+d_{3} \alpha_{23} \bar{u}_{3}\right. \\
& +d_{1}\left(d_{2}+\alpha_{21} \bar{u}_{1}+\alpha_{23} \bar{u}_{3}+d_{3}+\alpha_{32} \bar{u}_{2}\right) \\
& \left.+\alpha_{12} \bar{u}_{2}\left(d_{2}+\alpha_{23} \bar{u}_{3}+d_{3}+\alpha_{32} \bar{u}_{2}\right)\right] \zeta \\
& +\left(d_{1} d_{2}+d_{1} \alpha_{21} \bar{u}_{1}+d_{2} \alpha_{12} \bar{u}_{2}\right)\left(d_{3}+\alpha_{32} \bar{u}_{2}\right) \\
& +d_{3} \alpha_{23} \bar{u}_{3}\left(d_{1}+\alpha_{12} \bar{u}_{2}\right) \triangleq \widetilde{p}(\zeta) .
\end{aligned}
$$

By the Routh-Hurwitz criterion, the three roots $\zeta_{1}, \zeta_{2}$ and $\zeta_{3}$ of $\widetilde{p}(\zeta)=0$ all have negative real parts. Then we can conclude that there exists a positive constant $\widetilde{\delta}$ such that $\operatorname{Re}\left\{\zeta_{1}\right\}, \operatorname{Re}\left\{\zeta_{2}\right\}, \operatorname{Re}\left\{\zeta_{3}\right\} \leq-\widetilde{\delta}$. By continuity, we see that there exists $i_{0}$ such that the three roots of 
$\widetilde{p}_{i}(\zeta)=0$ satisfy $\operatorname{Re}\left\{\zeta_{i, 1}\right\}, \operatorname{Re}\left\{\zeta_{i, 2}\right\}, \operatorname{Re}\left\{\zeta_{i, 3}\right\} \leq-\widetilde{\delta} / 2$ for all $i \geq i_{0}$. Then

$$
\operatorname{Re}\left\{\mu_{i, 1}\right\}, \operatorname{Re}\left\{\mu_{i, 2}\right\}, \operatorname{Re}\left\{\mu_{i, 3}\right\} \leq-\frac{\lambda_{i} \widetilde{\delta}}{2} \leq-\frac{\widetilde{\delta}}{2}
$$

for all $i \geq i_{0}$. Let

$$
\delta=\min \left\{\frac{\widetilde{\delta}}{2}, \max _{1 \leq i \leq i_{0}}\left\{\operatorname{Re}\left\{\mu_{i, 1}\right\}, \operatorname{Re}\left\{\mu_{i, 2}\right\}, \operatorname{Re}\left\{\mu_{i, 3}\right\}\right\}\right\} .
$$

Then (2.7) holds true and we have the following results.

Proposition 2.2. Suppose that (2.2) and (2.6) hold true. Then the positive equilibrium $\overline{\mathbf{u}}$ of (1.1) is uniformly asymptotically stable.

It is difficult to obtain the global stability of positive equilibrium $\overline{\mathbf{u}}$ for cross-diffusion system (1.1). But, for the weakly coupled system of (1.1), the authors of [20] investigated the global stability of positive equilibrium. Their result, i.e., Theorem 2.2, shows that the positive equilibrium $\overline{\mathbf{u}}$ of (1.1) is globally asymptotically stable provided that $\alpha_{12}=\alpha_{21}=\alpha_{23}=\alpha_{32}=0$. So we have the following conclusion immediately.

Proposition 2.3. Let $\alpha_{12}=\alpha_{21}=\alpha_{23}=\alpha_{32}=0$. Then problem (1.1) has no non-constant positive steady state.

3. A priori bounds for positive steady states of PDEs. The corresponding steady state problem of (1.1) is

$$
\left\{\begin{array}{l}
-\Delta \mathbf{\Phi}(\mathbf{u})=\mathbf{F}(\mathbf{u}) \text { in } \Omega, \\
\frac{\partial \mathbf{u}}{\partial \nu}=0 \text { on } \partial \Omega .
\end{array}\right.
$$

In this section, we give a priori positive upper and lower bounds for positive solutions to the elliptic system (3.1). For this, we need the following two results.

Lemma 3.1. (Maximum principle (see [23])). Let $g(x, w) \in C(\Omega \times$ $\left.\mathbb{R}^{1}\right)$ and $b_{j}(x) \in C(\bar{\Omega}), j=1, \ldots, N$. 
(i) If $w \in C^{2}(\Omega) \cap C^{1}(\bar{\Omega})$ satisfies

$$
\left\{\begin{array}{l}
-\Delta w(x) \leq \sum_{j=1}^{N} b_{j}(x) w_{x_{j}}+g(x, w(x)) \quad \text { in } \Omega, \\
\frac{\partial w}{\partial \nu} \leq 0 \text { on } \partial \Omega
\end{array}\right.
$$

and $w\left(x_{0}\right)=\max _{\bar{\Omega}} w$, then $g\left(x_{0}, w\left(x_{0}\right)\right) \geq 0$.

(ii) If $w \in C^{2}(\Omega) \cap C^{1}(\bar{\Omega})$ satisfies

$$
\left\{\begin{array}{l}
-\Delta w(x) \geq \sum_{j=1}^{N} b_{j}(x) w_{x_{j}}+g(x, w(x)) \quad \text { in } \Omega, \\
\frac{\partial w}{\partial \nu} \geq 0 \quad \text { on } \partial \Omega,
\end{array}\right.
$$

and $w\left(x_{0}\right)=\min _{\bar{\Omega}} w$, then $g\left(x_{0}, w\left(x_{0}\right)\right) \leq 0$.

Lemma 3.2. (Harnack inequality (see [23])). Let $w \in C^{2}(\Omega) \cap C^{1}(\bar{\Omega})$ be a positive solution to $-\Delta w(x)=c(x) w(x)$ with $c \in C(\bar{\Omega})$, subject to a homogeneous Neumann boundary condition. Then there exists a positive constant $C=C\left(N, \Omega,\|c\|_{\infty}\right)$ such that

$$
\max _{\bar{\Omega}} w \leq C \min _{\bar{\Omega}} w
$$

In this paper, we assume that the classical solution is in $C^{2}(\Omega) \cap$ $C^{1}(\bar{\Omega})$. The results of upper and lower bounds can be stated as follows.

Proposition 3.3. (Upper bound). For any positive classical solution $\mathbf{u}$ of (3.1),

$$
\max _{\bar{\Omega}} u_{i} \leq M_{i}, \quad i=1,2,3
$$

where

$$
\begin{aligned}
& M_{1}=\frac{a_{1}\left(d_{1} b_{12}+a_{1} \alpha_{12}\right)}{d_{1} b_{11} b_{12}} \\
& M_{2}=\left(d_{2}+\alpha_{21} M_{1}+\frac{\alpha_{23}\left(a_{2}+b_{21} M_{1}\right)}{b_{23}}\right) \frac{a_{2}+b_{21} M_{1}}{d_{2} b_{22}}
\end{aligned}
$$

and

$$
M_{3}=\left(d_{3}+\alpha_{32} M_{2}\right) \frac{a_{3}+b_{32} M_{2}}{d_{3} b_{33}} .
$$


Proof. Problem (3.1) can be rewritten as

$$
\left\{\begin{array}{l}
-\Delta \phi_{1}=u_{1}\left(a_{1}-b_{11} u_{1}-b_{12} u_{2}\right) \quad \text { in } \Omega, \\
-\Delta \phi_{2}=u_{2}\left(a_{2}+b_{21} u_{1}-b_{22} u_{2}-b_{23} u_{3}\right) \quad \text { in } \Omega, \\
-\Delta \phi_{3}=u_{3}\left(a_{3}+b_{32} u_{2}-b_{33} u_{3}\right) \quad \text { in } \Omega, \\
\frac{\partial \phi_{1}}{\partial \nu}=\frac{\partial \phi_{2}}{\partial \nu}=\frac{\partial \phi_{3}}{\partial \nu}=0 \quad \text { on } \partial \Omega .
\end{array}\right.
$$

Let $x_{i} \in \bar{\Omega}$ be a point such that $\phi_{i}\left(x_{i}\right)=\max _{\bar{\Omega}} \phi_{i}$. Applying Lemma 3.1, we have

$$
u_{1}\left(x_{1}\right)\left(a_{1}-b_{11} u_{1}\left(x_{1}\right)-b_{12} u_{2}\left(x_{1}\right)\right) \geq 0 .
$$

Then, by the positivity of $\mathbf{u}$, one can obtain

$$
u_{1}\left(x_{1}\right) \leq \frac{a_{1}}{b_{11}}, \quad u_{2}\left(x_{1}\right) \leq \frac{a_{1}}{b_{12}}
$$

and

$$
\begin{aligned}
\max _{\bar{\Omega}} u_{1} & \leq \frac{1}{d_{1}} \max _{\bar{\Omega}} \phi_{1}=\frac{1}{d_{1}}\left(d_{1}+\alpha_{12} u_{2}\left(x_{1}\right)\right) u_{1}\left(x_{1}\right) \\
& \leq\left(d_{1}+\frac{\alpha_{12} a_{1}}{b_{12}}\right) \frac{a_{1}}{d_{1} b_{11}}
\end{aligned}
$$

Similarly, from the second equation in (3.2) and Lemma 3.1, we can obtain

$$
u_{2}\left(x_{2}\right) \leq \frac{a_{2}+b_{21} M_{1}}{b_{22}}, \quad u_{3}\left(x_{2}\right) \leq \frac{a_{2}+b_{21} M_{1}}{b_{23}}
$$

and

$$
\begin{aligned}
\max _{\bar{\Omega}} u_{2} & \leq \frac{1}{d_{2}} \max _{\bar{\Omega}} \phi_{2}=\frac{1}{d_{2}}\left(d_{2}+\alpha_{21} u_{1}\left(x_{2}\right)+\alpha_{23} u_{3}\left(x_{2}\right)\right) u_{2}\left(x_{2}\right) \\
& \leq\left(d_{2}+\alpha_{21} M_{1}+\frac{\alpha_{23}\left(a_{2}+b_{21} M_{1}\right)}{b_{23}}\right) \frac{a_{2}+b_{21} M_{1}}{d_{2} b_{22}}
\end{aligned}
$$

From Lemma 3.1 and the third equation in (3.2), we conclude that $u_{3}\left(x_{3}\right) \leq\left(a_{3}+b_{32} M_{2}\right) / b_{33}$. Thus,

$$
\begin{aligned}
\max _{\bar{\Omega}} u_{3} & \leq \frac{1}{d_{3}} \max _{\bar{\Omega}} \phi_{3}=\frac{1}{d_{3}}\left(d_{3}+\alpha_{32} u_{2}\left(x_{3}\right)\right) u_{3}\left(x_{3}\right) \\
& \leq\left(d_{3}+\alpha_{32} M_{2}\right) \frac{a_{3}+b_{32} M_{2}}{d_{3} b_{33}}
\end{aligned}
$$


This completes the proof.

Proposition 3.4. (Lower bound). Let $C_{3}=C_{3}\left(N, \Omega, d_{j}, a_{j}, b_{j j}, j=\right.$ $\left.1,2,3, \alpha_{12}, \alpha_{21}, \alpha_{23}, \alpha_{32}, b_{12}, b_{21}, b_{23}, b_{32}\right)$ be a given positive constant. Then there exist positive constants $m_{i}=m_{i}\left(N, \Omega, d_{j}, a_{j}, b_{j j}, j=\right.$ $\left.1,2,3, \alpha_{12}, \alpha_{21}, \alpha_{23}, \alpha_{32}, b_{12}, b_{21}, b_{23}, b_{32}\right), i=1,2,3$, such that, when $a_{3} b_{23}>a_{2} b_{33}\left(1+\left(\alpha_{32} / d_{3}\right) M_{2}\right) C_{3}$, the positive classical solution $\mathbf{u}$ of (3.1) satisfies

$$
\min _{\bar{\Omega}} u_{i} \geq m_{i}, \quad i=1,2,3
$$

Proof. Problem (3.1) is equivalent to

$$
\left\{\begin{array}{l}
-\Delta \phi_{1}=\frac{a_{1}-b_{11} u_{1}-b_{12} u_{2}}{d_{1}+\alpha_{12} u_{2}} \phi_{1} \quad \text { in } \Omega \\
-\Delta \phi_{2}=\frac{a_{2}+b_{21} u_{1}-b_{22} u_{2}-b_{23} u_{3}}{d_{2}+\alpha_{21} u_{1}+\alpha_{23} u_{3}} \phi_{2} \quad \text { in } \Omega \\
-\Delta \phi_{3}=\frac{a_{3}+b_{32} u_{2}-b_{33} u_{3}}{d_{3}+\alpha_{32} u_{2}} \phi_{3} \quad \text { in } \Omega \\
\frac{\partial \phi_{1}}{\partial \nu}=\frac{\partial \phi_{2}}{\partial \nu}=\frac{\partial \phi_{3}}{\partial \nu}=0 \quad \text { on } \partial \Omega
\end{array}\right.
$$

Since inequalities

$$
\begin{gathered}
\left\|\frac{a_{1}-b_{11} u_{1}-b_{12} u_{2}}{d_{1}+\alpha_{12} u_{2}}\right\|_{\infty} \leq \frac{a_{1}+b_{11} M_{1}+b_{12} M_{2}}{d_{1}}, \\
\left\|\frac{a_{2}+b_{21} u_{1}-b_{22} u_{2}-b_{23} u_{3}}{d_{2}+\alpha_{21} u_{1}+\alpha_{23} u_{3}}\right\|_{\infty} \leq \frac{a_{2}+b_{21} M_{1}+b_{22} M_{2}+b_{23} M_{3}}{d_{2}}, \\
\left\|\frac{a_{3}+b_{32} u_{2}-b_{33} u_{3}}{d_{3}+\alpha_{32} u_{2}}\right\|_{\infty} \leq \frac{a_{3}+b_{32} M_{2}+b_{33} M_{3}}{d_{3}}
\end{gathered}
$$

hold, the Harnack inequality in Lemma 3.2 shows that there exist positive constants $C_{i}=C_{i}\left(N, \Omega, d_{j}, a_{j}, b_{j j}, j=1,2,3, \alpha_{12}, \alpha_{21}, \alpha_{23}\right.$, $\left.\alpha_{32}, b_{12}, b_{21}, b_{23}, b_{32}\right), i=1,2,3$, such that

$$
\max _{\bar{\Omega}} \phi_{i} \leq C_{i} \min _{\bar{\Omega}} \phi_{i}, \quad i=1,2,3
$$

Thus,

$$
\left\{\begin{array}{l}
\frac{\max _{\bar{\Omega}} u_{1}}{\min _{\bar{\Omega}} u_{1}} \leq \frac{\max _{\bar{\Omega}} \phi_{1}}{\min _{\bar{\Omega}} \phi_{1}} \frac{d_{1}+\alpha_{12} \max _{\bar{\Omega}} u_{2}}{d_{1}+\alpha_{12} \min _{\bar{\Omega}} u_{2}} \leq C_{1}\left(1+\frac{\alpha_{12}}{d_{1}} M_{2}\right) \triangleq C_{4} \\
\frac{\max _{\bar{\Omega}} u_{2}}{\min _{\bar{\Omega}} u_{2}} \leq \frac{\max _{\bar{\Omega}} \phi_{2}}{\min _{\bar{\Omega}} \phi_{2}} \frac{d_{2}+\alpha_{21} \max _{\bar{\Omega}} u_{1}+\alpha_{23} \max _{\bar{\Omega}} u_{3}}{d_{2}+\alpha_{21} \min _{\bar{\Omega}} u_{1}+\alpha_{23} \min _{\bar{\Omega}} u_{3}} \\
\leq C_{2}\left(1+\frac{\alpha_{21}}{d_{2}} M_{1}+\frac{\alpha_{23}}{d_{2}} M_{3}\right) \triangleq C_{5} \\
\frac{\max _{\bar{\Omega}} u_{3}}{\min _{\bar{\Omega}} u_{3}} \leq \frac{\max _{\bar{\Omega}} \phi_{3}}{\min _{\bar{\Omega}} \phi_{3}} \frac{d_{3}+\alpha_{32} \max _{\bar{\Omega}} u_{2}}{d_{3}+\alpha_{32} \min _{\bar{\Omega}} u_{2}} \leq C_{3}\left(1+\frac{\alpha_{32}}{d_{3}} M_{2}\right) \triangleq C_{6}
\end{array}\right.
$$


On the other hand, by integrating the third equation in (3.2), we have

$$
\int_{\Omega} u_{3}\left(a_{3}+b_{32} u_{2}-b_{33} u_{3}\right) d x=0
$$

Then there exists a point $z_{3} \in \Omega$ such that $a_{3}+b_{32} u_{2}\left(z_{3}\right)-b_{33} u_{3}\left(z_{3}\right)=$ 0 , which implies that $u_{3}\left(z_{3}\right) \geq a_{3} / b_{33}$ and

$$
\min _{\bar{\Omega}} u_{3} \geq \frac{1}{C_{6}} \max _{\bar{\Omega}} u_{3} \geq \frac{1}{C_{6}} u_{3}\left(z_{3}\right) \geq \frac{a_{3}}{C_{6} b_{33}} \triangleq m_{3} .
$$

Similarly, by integrating the second equation in (3.2), we obtain

$$
\int_{\Omega} u_{2}\left(a_{2}+b_{21} u_{1}-b_{22} u_{2}-b_{23} u_{3}\right) d x=0
$$

Then there exists a point $z_{2} \in \Omega$ such that $a_{2}+b_{21} u_{1}\left(z_{2}\right)-b_{22} u_{2}\left(z_{2}\right)-$ $b_{23} u_{3}\left(z_{2}\right)=0$. It follows then that

$$
\begin{aligned}
u_{1}\left(z_{2}\right) & >\frac{b_{23} u_{3}\left(z_{2}\right)-a_{2}}{b_{21}}>\frac{b_{23} m_{3}-a_{2}}{b_{21}} \\
& =\frac{1}{b_{21}}\left[\frac{a_{3} b_{23}}{b_{33}\left(1+\left(\alpha_{32} / d_{3}\right) M_{2}\right) C_{3}}-a_{2}\right] \triangleq m_{1}^{*} .
\end{aligned}
$$

So

$$
\min _{\bar{\Omega}} u_{1} \geq \frac{1}{C_{4}} \max _{\bar{\Omega}} u_{1} \geq \frac{1}{C_{4}} u_{1}\left(z_{2}\right)>\frac{m_{1}^{*}}{C_{4}} \triangleq m_{1} .
$$

Now we need to estimate the positive lower bound of $u_{2}$. Denote $M_{2}^{*}=\max _{\bar{\Omega}} u_{2}$. Let $y_{0}, z_{0} \in \bar{\Omega}$ be two points such that $\phi_{3}\left(y_{0}\right)=$ $\max _{\bar{\Omega}} \phi_{3}$ and $\phi_{3}\left(z_{0}\right)=\min _{\bar{\Omega}} \phi_{3}$. Applying Lemma 3.1 to the third equation in (3.3), we have

$$
a_{3}+b_{32} u_{2}\left(y_{0}\right)-b_{33} u_{3}\left(y_{0}\right) \geq 0 \geq a_{3}+b_{32} u_{2}\left(z_{0}\right)-b_{33} u_{3}\left(z_{0}\right) .
$$

This implies that

$$
u_{3}\left(y_{0}\right) \leq \frac{a_{3}+b_{32} M_{2}^{*}}{b_{33}}, \quad u_{3}\left(z_{0}\right) \geq \frac{a_{3}}{b_{33}},
$$

and

$$
\frac{\max _{\bar{\Omega}} u_{3}}{\min _{\bar{\Omega}} u_{3}} \leq \frac{\left(d_{3}+\alpha_{32} \max _{\bar{\Omega}} u_{2}\right) \max _{\bar{\Omega}} u_{3}}{\left(d_{3}+\alpha_{32} \min _{\bar{\Omega}} u_{2}\right) \min _{\bar{\Omega}} u_{3}} \frac{\max _{\bar{\Omega}} \phi_{3}}{\min _{\bar{\Omega}} \phi_{3}}
$$




$$
\begin{aligned}
& \leq\left(1+\frac{\alpha_{32}}{d_{3}} M_{2}^{*}\right) \frac{\left(d_{3}+\alpha_{32} u_{2}\left(y_{0}\right)\right) u_{3}\left(y_{0}\right)}{\left(d_{3}+\alpha_{32} u_{2}\left(z_{0}\right)\right) u_{3}\left(z_{0}\right)} \\
& \leq\left(1+\frac{\alpha_{32}}{d_{3}} M_{2}^{*}\right)^{2} \frac{a_{3}+b_{32} M_{2}^{*}}{a_{3}}
\end{aligned}
$$

Hence, there exists a positive constant $\epsilon>0$ such that $M_{2}^{*}>\epsilon$, which, combining with (3.4), indicates that there exists a positive constant $m_{2}$ such that $\min _{\bar{\Omega}} u_{2} \geq m_{2}$.

Remark 3.5. According to [32], [9, Theorem 8.20] and [23, Lemma $2.2]$, we know that $C=C\left(N, \Omega,\|c\|_{\infty}\right)$ in Lemma 3.2 is monotone increasing with respect to $\|c\|_{\infty}$. Fix parameters in system (3.1) except for $b_{23}$. If $b_{23}$ increases, then $M_{2}$ and $\left(a_{3}+b_{32} M_{2}+b_{33} M_{3}\right) / d_{3}$ both decrease, and then $C_{3}$ decreases. Therefore, a large enough value of $b_{23}$ can ensure the inequality

$$
a_{3} b_{23}>a_{2} b_{33}\left(1+\frac{\alpha_{32}}{d_{3}} M_{2}\right) C_{3} .
$$

4. Existence of non-constant positive steady states. In this section, we shall use a Leray-Schauder degree theory to develop a general setting to establish the existence of stationary patterns for system (1.1). Denote

$$
\begin{aligned}
\mathbf{X}^{+} & =\{\mathbf{u} \in \mathbf{X} \mid \mathbf{u}>\mathbf{0} \text { on } \bar{\Omega}\}, \\
B(M) & =\left\{\mathbf{u} \in \mathbf{X}^{+} \mid M^{-1}<u_{i}<M \text { on } \bar{\Omega}, i=1,2,3\right\} .
\end{aligned}
$$

As in Section 2, we denote the constant positive equilibrium of system (1.1) by $\overline{\mathbf{u}}$.

Since the determinant $\operatorname{det} \boldsymbol{\Phi}_{\mathbf{u}}(\mathbf{u})$ is positive for all non-negative $\mathbf{u}$, $\left[\boldsymbol{\Phi}_{\mathbf{u}}(\overline{\mathbf{u}})\right]^{-1}$ exists and $\operatorname{det}\left\{\left[\boldsymbol{\Phi}_{\mathbf{u}}(\overline{\mathbf{u}})\right]^{-1}\right\}$ is positive. Thus, $\mathbf{u}$ is a positive solution of system (3.1) if and only if

$$
\begin{aligned}
\Psi(\mathbf{u}) \triangleq & \mathbf{u}-(\mathbf{I}-\Delta)^{-1}\left\{\left[\boldsymbol{\Phi}_{\mathbf{u}}(\mathbf{u})\right]^{-1}[\mathbf{F}(\mathbf{u})\right. \\
& \left.\left.+\nabla \mathbf{u} \boldsymbol{\Phi}_{\mathbf{u u}}(\mathbf{u}) \nabla \mathbf{u}\right]+\mathbf{u}\right\}=0 \quad \text { in } \mathbf{X}^{+}
\end{aligned}
$$

where $(\mathbf{I}-\Delta)^{-1}$ is the inverse of $\mathbf{I}-\Delta$ in $\mathbf{X}$, subject to the homogeneous Neumann boundary condition. Since $\Psi(\cdot)$ is a compact perturbation of the identity operator, the Leray-Schauder degree $\operatorname{deg}(\Psi(\cdot), 0, B(M))$ is 
well defined if $\Psi(\mathbf{u}) \neq 0$ for any $\mathbf{u} \in \partial B(M)$. Further, an elementary calculation shows

$$
\mathrm{D}_{\mathbf{u}} \Psi(\overline{\mathbf{u}})=\mathbf{I}-(\mathbf{I}-\Delta)^{-1}\left\{\left[\boldsymbol{\Phi}_{\mathbf{u}}(\overline{\mathbf{u}})\right]^{-1} \mathbf{F}_{\mathbf{u}}(\overline{\mathbf{u}})+\mathbf{I}\right\} \quad \text { in } \mathcal{L}(\mathbf{X}, \mathbf{X}) .
$$

We recall that, if $\mathrm{D}_{\mathbf{u}} \Psi$ does not have any pure imaginary or zero eigenvalue, the index of $\Psi$ at the fixed point $\mathbf{u}^{*}$ is defined as index $\left(\Psi(\cdot), \mathbf{u}^{*}\right)=$ $(-1)^{r}$, where $r$ is the total number of eigenvalues of $\mathrm{D}_{\mathbf{u}} \Psi$ with negative real parts (counting multiplicities). Then the degree deg $(\Psi(\cdot), 0, B(M))$ is equal to the sum of the indexes over all solutions to $\Psi=0$ in $B(M)$, provided that $\Psi \neq 0$ on $\partial B(M)$.

In order to calculate $r$, we employ the eigenspaces of $-\Delta$. Using the decomposition (2.5) we investigate the eigenvalues of $\mathrm{D}_{\mathbf{u}} \Psi(\overline{\mathbf{u}})$. First, we know $\mathbf{X}_{i j}$ is invariant under $\mathrm{D}_{\mathbf{u}} \Psi(\overline{\mathbf{u}})$ for each $i \in \mathbb{N}$ and each $j \in\left[1, \operatorname{dim} E\left(\lambda_{i}\right)\right] \cap \mathbb{N}$, i.e., $\mathrm{D}_{\mathbf{u}} \Psi(\overline{\mathbf{u}}) \mathbf{u} \in \mathbf{X}_{i j}$ for any $\mathbf{u} \in \mathbf{X}_{i j}$. Hence, $\mu$ is an eigenvalue of $\mathrm{D}_{\mathbf{u}} \Psi(\overline{\mathbf{u}})$ on $\mathbf{X}_{i j}$ if and only if it is an eigenvalue of the matrix

$$
\mathbf{I}-\frac{1}{1+\lambda_{i}}\left\{\left[\boldsymbol{\Phi}_{\mathbf{u}}(\overline{\mathbf{u}})\right]^{-1} \mathbf{F}_{\mathbf{u}}(\overline{\mathbf{u}})+\mathbf{I}\right\}=\frac{1}{1+\lambda_{i}}\left\{\lambda_{i} \mathbf{I}-\left[\boldsymbol{\Phi}_{\mathbf{u}}(\overline{\mathbf{u}})\right]^{-1} \mathbf{F}_{\mathbf{u}}(\overline{\mathbf{u}})\right\} .
$$

Obviously, $\operatorname{det}\left\{\mathbf{F}_{\mathbf{u}}(\overline{\mathbf{u}})\right\}<0$ under the condition (2.2). So $\mathrm{D}_{\mathbf{u}} \Psi(\overline{\mathbf{u}})$ is invertible if and only if, for any $i \geq 1$, the matrix

$$
\frac{1}{1+\lambda_{i}}\left\{\lambda_{i} \mathbf{I}-\left[\boldsymbol{\Phi}_{\mathbf{u}}(\overline{\mathbf{u}})\right]^{-1} \mathbf{F}_{\mathbf{u}}(\overline{\mathbf{u}})\right\}
$$

is non-singular. Denote

$$
H(\lambda) \triangleq H(\overline{\mathbf{u}}, \lambda)=\operatorname{det}\left\{\lambda \mathbf{I}-\left[\mathbf{\Phi}_{\mathbf{u}}(\overline{\mathbf{u}})\right]^{-1} \mathbf{F}_{\mathbf{u}}(\overline{\mathbf{u}})\right\}
$$

We notice that, if $H\left(\lambda_{i}\right) \neq 0$, then for each $j \in\left[1, \operatorname{dim} E\left(\lambda_{i}\right)\right]$, the number of negative eigenvalues of $\mathrm{D}_{\mathbf{u}} \Psi(\overline{\mathbf{u}})$ on $\mathbf{X}_{i j}$ is odd if and only if $H\left(\lambda_{i}\right)<0$. In conclusion, we have the following result.

Proposition 4.1. Assume that, for each $i \geq 1$, the matrix $\lambda_{i} \mathbf{I}-$ $\left[\mathbf{\Phi}_{\mathbf{u}}(\overline{\mathbf{u}})\right]^{-1} \mathbf{F}_{\mathbf{u}}(\overline{\mathbf{u}})$ is non-singular. Then

$$
\operatorname{index}(\Psi(\cdot), \overline{\mathbf{u}})=(-1)^{\sigma}, \quad \text { where } \quad \sigma=\sum_{\substack{i \geq 1 \\ H\left(\lambda_{i}\right)<0}} \operatorname{dim} E\left(\lambda_{i}\right) .
$$


According to the above proposition, we should consider the sign of $H\left(\lambda_{i}\right)$ in order to calculate index $(\Psi(\cdot), \overline{\mathbf{u}})$. Since

$$
H(\lambda)=\operatorname{det}\left\{\left[\boldsymbol{\Phi}_{\mathbf{u}}(\overline{\mathbf{u}})\right]^{-1}\right\} \operatorname{det}\left\{\lambda \boldsymbol{\Phi}_{\mathbf{u}}(\overline{\mathbf{u}})-\mathbf{F}_{\mathbf{u}}(\overline{\mathbf{u}})\right\}
$$

and $\operatorname{det}\left\{\left[\boldsymbol{\Phi}_{\mathbf{u}}(\overline{\mathbf{u}})\right]^{-1}\right\}>0$, we only need to consider the sign of $\operatorname{det}\left\{\lambda \boldsymbol{\Phi}_{\mathbf{u}}(\overline{\mathbf{u}})-\mathbf{F}_{\mathbf{u}}(\overline{\mathbf{u}})\right\}$. A direct calculation shows

$$
\begin{aligned}
\operatorname{det}\left\{\lambda \boldsymbol{\Phi}_{\mathbf{u}}(\overline{\mathbf{u}})-\mathbf{F}_{\mathbf{u}}(\overline{\mathbf{u}})\right\}= & A_{3}\left(\alpha_{21}, \alpha_{32}\right) \lambda^{3}+A_{2}\left(\alpha_{21}, \alpha_{32}\right) \lambda^{2} \\
& +A_{1}\left(\alpha_{21}, \alpha_{32}\right) \lambda-\operatorname{det}\left\{\mathbf{F}_{\mathbf{u}}(\overline{\mathbf{u}})\right\} \\
\triangleq & q\left(\alpha_{21}, \alpha_{32} ; \lambda\right),
\end{aligned}
$$

where

$$
\begin{aligned}
A_{3}\left(\alpha_{21}, \alpha_{32}\right)= & \left(d_{1}+\alpha_{12} \bar{u}_{2}\right)\left(d_{2} d_{3}+d_{2} \alpha_{32} \bar{u}_{2}+d_{3} \alpha_{23} \bar{u}_{3}\right) \\
& +d_{1} \alpha_{21} \bar{u}_{1}\left(d_{3}+\alpha_{32} \bar{u}_{2}\right)>0, \\
A_{2}\left(\alpha_{21}, \alpha_{32}\right)= & d_{3} b_{11} \bar{u}_{1}\left(d_{2}+\alpha_{21} \bar{u}_{1}+\alpha_{23} \bar{u}_{3}\right)+b_{11} \alpha_{32} \bar{u}_{1} \bar{u}_{2}\left(d_{2}+\alpha_{21} \bar{u}_{1}\right) \\
& +b_{33} \alpha_{12} \bar{u}_{2} \bar{u}_{3}\left(d_{2}+\alpha_{23} \bar{u}_{3}\right)+d_{1} b_{33} \bar{u}_{3}\left(d_{2}+\alpha_{21} \bar{u}_{1}+\alpha_{23} \bar{u}_{3}\right) \\
& +b_{22} \bar{u}_{2}\left(d_{1}+\alpha_{12} \bar{u}_{2}\right)\left(d_{3}+\alpha_{32} \bar{u}_{2}\right) \\
& +\left(\alpha_{23} b_{32}-\alpha_{32} b_{23}\right) \bar{u}_{2} \bar{u}_{3}\left(d_{1}+\alpha_{12} \bar{u}_{2}\right) \\
& +\left(\alpha_{12} b_{21}-\alpha_{21} b_{12}\right) \bar{u}_{1} \bar{u}_{2}\left(d_{3}+\alpha_{32} \bar{u}_{2}\right), \\
A_{1}\left(\alpha_{21}, \alpha_{32}\right)= & b_{11} b_{33} \bar{u}_{1} \bar{u}_{3}\left(d_{2}+\alpha_{21} \bar{u}_{1}+\alpha_{23} \bar{u}_{3}\right)+\left(b_{22} b_{33}+b_{23} b_{32}\right) \bar{u}_{2} \bar{u}_{3} \\
\cdot & \left(d_{1}+\alpha_{12} \bar{u}_{2}\right)+\left(b_{11} b_{22}+b_{12} b_{21}\right) \bar{u}_{1} \bar{u}_{2}\left(d_{3}+\alpha_{32} \bar{u}_{2}\right) \\
& +\left[\left(\alpha_{23} b_{32}-\alpha_{32} b_{23}\right) b_{11}+\left(\alpha_{12} b_{21}-\alpha_{21} b_{12}\right) b_{33}\right] \bar{u}_{1} \bar{u}_{2} \bar{u}_{3} .
\end{aligned}
$$

Let $\bar{\lambda}_{1}, \bar{\lambda}_{2}$ and $\bar{\lambda}_{3}$ be the three roots of $q\left(\alpha_{21}, \alpha_{32} ; \lambda\right)=0$ with $\operatorname{Re}\left\{\bar{\lambda}_{1}\right\} \leq \operatorname{Re}\left\{\bar{\lambda}_{2}\right\} \leq \operatorname{Re}\left\{\bar{\lambda}_{3}\right\}$. Then

$$
\bar{\lambda}_{1} \bar{\lambda}_{2} \bar{\lambda}_{3}=\frac{\operatorname{det}\left\{\mathbf{F}_{\mathbf{u}}(\overline{\mathbf{u}})\right\}}{A_{3}\left(\alpha_{21}, \alpha_{32}\right)}=-\frac{\left(b_{11} b_{22} b_{33}+b_{11} b_{23} b_{32}+b_{12} b_{21} b_{33}\right) \bar{u}_{1} \bar{u}_{2} \bar{u}_{3}}{A_{3}\left(\alpha_{21}, \alpha_{32}\right)}<0 .
$$

Notice that $A_{3}>0$. So one of $\bar{\lambda}_{1}, \bar{\lambda}_{2}, \bar{\lambda}_{3}$ is real and negative, and the product of the other two is positive.

Now we consider the dependence of $q\left(\alpha_{21}, \alpha_{32} ; \lambda\right)$ on $\alpha_{21}$. Perform the following limits:

$$
\lim _{\alpha_{21} \rightarrow \infty} \frac{A_{3}\left(\alpha_{21}, \alpha_{32}\right)}{\alpha_{21}}=d_{1}\left(d_{3}+\alpha_{32} \bar{u}_{2}\right) \bar{u}_{1} \triangleq \Lambda_{3}\left(\alpha_{32}\right)>0,
$$




$$
\begin{gathered}
\lim _{\alpha_{21} \rightarrow \infty} \frac{A_{2}\left(\alpha_{21}, \alpha_{32}\right)}{\alpha_{21}}=b_{11}\left(d_{3}+\alpha_{32} \bar{u}_{2}\right) \bar{u}_{1}^{2}+d_{1} b_{33} \bar{u}_{1} \bar{u}_{3} \\
-b_{12}\left(d_{3}+\alpha_{32} \bar{u}_{2}\right) \bar{u}_{1} \bar{u}_{2} \triangleq \Lambda_{2}\left(\alpha_{32}\right), \\
\lim _{\alpha_{21} \rightarrow \infty} \frac{A_{1}\left(\alpha_{21}, \alpha_{32}\right)}{\alpha_{21}}=b_{33}\left(b_{11} \bar{u}_{1}-b_{12} \bar{u}_{2}\right) \bar{u}_{1} \bar{u}_{3} \triangleq \Lambda_{1},
\end{gathered}
$$

and

$$
\lim _{\alpha_{21} \rightarrow \infty} \frac{q\left(\alpha_{21}, \alpha_{32} ; \lambda\right)}{\alpha_{21}}=\lambda\left[\Lambda_{3}\left(\alpha_{32}\right) \lambda^{2}+\Lambda_{2}\left(\alpha_{32}\right) \lambda+\Lambda_{1}\right] .
$$

If the parameters $a_{i}, b_{i j}$ of reaction terms satisfy

(4.2) $b_{11}\left(a_{1} b_{22} b_{33}+a_{1} b_{23} b_{32}+2 a_{3} b_{12} b_{23}\right)<b_{33}\left(a_{1} b_{12} b_{21}+2 a_{2} b_{11} b_{12}\right)$,

then $\Lambda_{1}<0$, and we have the following result.

Proposition 4.2. Suppose that (2.2) and (4.2) hold true. Then there exists a positive constant $\alpha_{21}^{*}$, such that for $\alpha_{21} \geq \alpha_{21}^{*}$, the three roots $\bar{\lambda}_{1}\left(\alpha_{21}\right), \bar{\lambda}_{2}\left(\alpha_{21}\right)$ and $\bar{\lambda}_{3}\left(\alpha_{21}\right)$ of $q\left(\alpha_{21}, \alpha_{32} ; \lambda\right)=0$ are all real and satisfy

$$
\left\{\begin{array}{l}
\lim _{\alpha_{21} \rightarrow \infty} \bar{\lambda}_{1}\left(\alpha_{21}\right)=\frac{-\Lambda_{2}-\sqrt{\Lambda_{2}^{2}-4 \Lambda_{1} \Lambda_{3}}}{2 \Lambda_{3}}<0, \\
\lim _{\alpha_{21} \rightarrow \infty} \bar{\lambda}_{2}\left(\alpha_{21}\right)=0, \\
\lim _{\alpha_{21} \rightarrow \infty} \bar{\lambda}_{3}\left(\alpha_{21}\right)=\frac{-\Lambda_{2}+\sqrt{\Lambda_{2}^{2}-4 \Lambda_{1} \Lambda_{3}}}{2 \Lambda_{3}} \triangleq \bar{\lambda}>0 .
\end{array}\right.
$$

Moreover, we can conclude that

$$
\left\{\begin{array}{l}
-\infty<\bar{\lambda}_{1}\left(\alpha_{21}\right)<0<\bar{\lambda}_{2}\left(\alpha_{21}\right)<\bar{\lambda}_{3}\left(\alpha_{21}\right) \\
q\left(\alpha_{21}, \alpha_{32} ; \lambda\right)<0 \text { if } \lambda \in\left(-\infty, \bar{\lambda}_{1}\left(\alpha_{21}\right)\right) \cup\left(\bar{\lambda}_{2}\left(\alpha_{21}\right), \bar{\lambda}_{3}\left(\alpha_{21}\right)\right), \\
q\left(\alpha_{21}, \alpha_{32} ; \lambda\right)>0 \text { if } \lambda \in\left(\bar{\lambda}_{1}\left(\alpha_{21}\right), \bar{\lambda}_{2}\left(\alpha_{21}\right)\right) \cup\left(\bar{\lambda}_{3}\left(\alpha_{21}\right), \infty\right) .
\end{array}\right.
$$

Next we discuss the dependence of $q\left(\alpha_{21}, \alpha_{32} ; \lambda\right)$ on $\alpha_{32}$. We have the following limits:

$$
\begin{aligned}
& \lim _{\alpha_{32} \rightarrow \infty} \frac{A_{3}\left(\alpha_{21}, \alpha_{32}\right)}{\alpha_{32}}=d_{2} \bar{u}_{2}\left(d_{1}+\alpha_{12} \bar{u}_{2}\right)+d_{1} \alpha_{21} \bar{u}_{1} \bar{u}_{2} \triangleq \widetilde{\Lambda}_{3}\left(\alpha_{21}\right), \\
& \lim _{\alpha_{32} \rightarrow \infty} \frac{A_{2}\left(\alpha_{21}, \alpha_{32}\right)}{\alpha_{32}}=b_{11}\left(d_{2}+\alpha_{21} \bar{u}_{1}\right) \bar{u}_{1} \bar{u}_{2}+b_{22}\left(d_{1}+\alpha_{12} \bar{u}_{2}\right) \bar{u}_{2}^{2} \\
& -b_{23}\left(d_{1}+\alpha_{12} \bar{u}_{2}\right) \bar{u}_{2} \bar{u}_{3} \triangleq \widetilde{\Lambda}_{2}\left(\alpha_{21}\right) \text {, }
\end{aligned}
$$




$$
\lim _{\alpha_{32} \rightarrow \infty} \frac{A_{1}\left(\alpha_{21}, \alpha_{32}\right)}{\alpha_{32}}=\left[\left(b_{11} b_{22}+b_{12} b_{21}\right) \bar{u}_{2}-b_{11} b_{23} \bar{u}_{3}\right] \bar{u}_{1} \bar{u}_{2} \triangleq \widetilde{\Lambda}_{1},
$$

and

$$
\lim _{\alpha_{32} \rightarrow \infty} \frac{q\left(\alpha_{21}, \alpha_{32} ; \lambda\right)}{\alpha_{32}}=\lambda\left[\widetilde{\Lambda}_{3}\left(\alpha_{21}\right) \lambda^{2}+\widetilde{\Lambda}_{2}\left(\alpha_{21}\right) \lambda+\widetilde{\Lambda}_{1}\right] .
$$

Similarly, under the condition

$$
\begin{aligned}
\left(b_{11} b_{22}\right. & \left.+b_{12} b_{21}\right)\left(a_{1} b_{21} b_{33}+a_{2} b_{11} b_{33}\right) \\
& <b_{11} b_{23}\left(a_{1} b_{21} b_{32}+a_{2} b_{11} b_{32}+2 a_{3} b_{11} b_{22}+2 a_{3} b_{12} b_{21}\right)
\end{aligned}
$$

we can acquire $\widetilde{\Lambda}_{1}<0$ and the following results.

Proposition 4.3. Suppose that (2.2) and (4.5) hold true. Then there exists a positive constant $\alpha_{32}^{*}$ such that, for $\alpha_{32} \geq \alpha_{32}^{*}$, the three roots $\bar{\lambda}_{1}\left(\alpha_{32}\right), \bar{\lambda}_{2}\left(\alpha_{32}\right), \bar{\lambda}_{3}\left(\alpha_{32}\right)$ of $q\left(\alpha_{21}, \alpha_{32} ; \lambda\right)=0$ are all real and satisfy

$$
\left\{\begin{array}{l}
\lim _{\alpha_{32} \rightarrow \infty} \bar{\lambda}_{1}\left(\alpha_{32}\right)=\frac{-\widetilde{\Lambda}_{2}-\sqrt{\widetilde{\Lambda}_{2}^{2}-4 \widetilde{\Lambda}_{1} \widetilde{\Lambda}_{3}}}{2 \widetilde{\Lambda}_{3}}<0, \\
\lim _{\alpha_{32} \rightarrow \infty} \bar{\lambda}_{2}\left(\alpha_{32}\right)=0, \\
\lim _{\alpha_{32} \rightarrow \infty} \bar{\lambda}_{3}\left(\alpha_{32}\right)=\frac{-\widetilde{\Lambda}_{2}+\sqrt{\widetilde{\Lambda}_{2}^{2}-4 \widetilde{\Lambda}_{1} \widetilde{\Lambda}_{3}}}{2 \widetilde{\Lambda}_{3}} \triangleq \bar{\lambda}^{\prime}>0 .
\end{array}\right.
$$

Moreover, we can conclude that

$$
\left\{\begin{array}{l}
-\infty<\bar{\lambda}_{1}\left(\alpha_{32}\right)<0<\bar{\lambda}_{2}\left(\alpha_{32}\right)<\bar{\lambda}_{3}\left(\alpha_{32}\right), \\
q\left(\alpha_{21}, \alpha_{32} ; \lambda\right)<0 \text { if } \lambda \in\left(-\infty, \bar{\lambda}_{1}\left(\alpha_{32}\right)\right) \cup\left(\bar{\lambda}_{2}\left(\alpha_{32}\right), \bar{\lambda}_{3}\left(\alpha_{32}\right)\right), \\
q\left(\alpha_{21}, \alpha_{32} ; \lambda\right)>0 \text { if } \lambda \in\left(\bar{\lambda}_{1}\left(\alpha_{32}\right), \bar{\lambda}_{2}\left(\alpha_{32}\right)\right) \cup\left(\bar{\lambda}_{3}\left(\alpha_{32}\right), \infty\right) .
\end{array}\right.
$$

Now we establish the global existence of non-constant positive solution to (1.1) with respect to the cross-diffusion coefficients $\alpha_{21}$ and $\alpha_{32}$, respectively, as the other parameters are all fixed positive constants. Our results are as follows.

Theorem 4.4. Assume that parameters $d_{i}, a_{i}, b_{i i}, i=1,2,3, \alpha_{12}, \alpha_{23}$, $\alpha_{32}, b_{12}, b_{21}$ and $b_{32}$ are all fixed, and satisfy (2.2) and (4.2). Let $\bar{\lambda}$ be given by the limit (4.3). If $\bar{\lambda} \in\left(\lambda_{n}, \lambda_{n+1}\right)$ for some $n \geq 1$, and the sum $\sigma_{n}=\sum_{i=1}^{n} \operatorname{dim} E\left(\lambda_{i}\right)$ is odd, then there exists a positive constant $\alpha_{21}^{*}$ such that problem (1.1) has at least one non-constant positive steady 
state provided that $\alpha_{21}>\alpha_{21}^{*}$ and

$$
a_{3} b_{23}>a_{2} b_{33}\left(1+\frac{\alpha_{32}}{d_{3}} M_{2}\right) C_{3}
$$

are fulfilled.

Proof. By Proposition 4.2, there exists a positive constant $\alpha_{21}^{*}$ such that, if $\alpha_{21}>\alpha_{21}^{*},(4.3)$ holds and

$$
\bar{\lambda}_{1}\left(\alpha_{21}\right)<0=\lambda_{1}<\bar{\lambda}_{2}\left(\alpha_{21}\right)<\lambda_{2}, \quad \bar{\lambda}_{3}\left(\alpha_{21}\right) \in\left(\lambda_{n}, \lambda_{n+1}\right) .
$$

For $t \in[0,1]$, define

$$
\begin{array}{rr}
d_{i}(t) \equiv d_{i}, \quad \alpha_{12}(t)=t \alpha_{12}, & \alpha_{21}(t)=t \alpha_{21}, \\
\alpha_{23}(t)=t \alpha_{23}, & \alpha_{32}(t)=t \alpha_{32}
\end{array}
$$

and

$$
\begin{aligned}
\boldsymbol{\Phi}(t ; \mathbf{u})= & \left(\phi_{1}(t ; \mathbf{u}), \phi_{2}(t ; \mathbf{u}), \phi_{3}(t ; \mathbf{u})\right)^{\mathrm{T}} \\
= & \left(\left(d_{1}(t)+\alpha_{12}(t) u_{2}\right) u_{1},\left(d_{2}(t)+\alpha_{21}(t) u_{1}+\alpha_{23}(t) u_{3}\right) u_{2},\right. \\
& \left.\left(d_{3}(t)+\alpha_{32}(t) u_{2}\right) u_{3}\right)^{\mathrm{T}},
\end{aligned}
$$

and then consider the problem

$$
\left\{\begin{array}{l}
-\Delta \mathbf{\Phi}(t ; \mathbf{u})=\mathbf{F}(\mathbf{u}) \text { in } \Omega \\
\frac{\partial \mathbf{u}}{\partial \nu}=0 \text { on } \partial \Omega
\end{array}\right.
$$

Then $\mathbf{u}$ is a non-constant positive steady state of (1.1) if and only if it is a non-constant positive solution of problem (4.8) for $t=1$. It is obvious that $\overline{\mathbf{u}}$ is the unique constant positive solution of (4.8) for any $t \in[0,1]$. From (4.1), we know that for any $t \in[0,1], \mathbf{u}$ is a positive solution of problem (4.8) if and only if

$$
\begin{array}{r}
\Psi(t ; \mathbf{u}) \triangleq \mathbf{u}-(\mathbf{I}-\Delta)^{-1}\left\{\left[\boldsymbol{\Phi}_{\mathbf{u}}(t ; \mathbf{u})\right]^{-1}\left[\mathbf{F}(\mathbf{u})+\nabla \mathbf{u} \boldsymbol{\Phi}_{\mathbf{u u}}(t ; \mathbf{u}) \nabla \mathbf{u}\right]+\mathbf{u}\right\}=0 \\
\text { in } \mathbf{X}^{+}
\end{array}
$$

It is evident that $\Psi(1 ; \mathbf{u})=\Psi(\mathbf{u})$. Proposition 4.1 indicates that $\Psi(0 ; \mathbf{u})=0$ only has constant positive solution $\overline{\mathbf{u}}$ in $\mathbf{X}^{+}$. A direct calculation shows that

$$
\mathrm{D}_{\mathbf{u}} \Psi=\mathbf{I}-(\mathbf{I}-\Delta)^{-1}\left\{\left[\boldsymbol{\Phi}_{\mathbf{u}}(t ; \overline{\mathbf{u}})\right]^{-1} \mathbf{F}_{\mathbf{u}}(\overline{\mathbf{u}})+\mathbf{I}\right\} .
$$


In particular,

$$
\begin{aligned}
& \mathrm{D}_{\mathbf{u}} \Psi(0 ; \overline{\mathbf{u}})=\mathbf{I}-(\mathbf{I}-\Delta)^{-1}\left\{\left[\widehat{\boldsymbol{\Phi}}_{\mathbf{u}}(\overline{\mathbf{u}})\right]^{-1} \mathbf{F}_{\mathbf{u}}(\overline{\mathbf{u}})+\mathbf{I}\right\} \\
& \mathrm{D}_{\mathbf{u}} \Psi(1 ; \overline{\mathbf{u}})=\mathbf{I}-(\mathbf{I}-\Delta)^{-1}\left\{\left[\boldsymbol{\Phi}_{\mathbf{u}}(\overline{\mathbf{u}})\right]^{-1} \mathbf{F}_{\mathbf{u}}(\overline{\mathbf{u}})+\mathbf{I}\right\}=\mathrm{D}_{\mathbf{u}} \Psi(\overline{\mathbf{u}}) .
\end{aligned}
$$

Here $\widehat{\boldsymbol{\Phi}}_{\mathbf{u}}(\overline{\mathbf{u}})=\left(\begin{array}{ccc}d_{1} & 0 & 0 \\ 0 & d_{2} & 0 \\ 0 & 0 & d_{3}\end{array}\right)$. Moreover, we already know that

$$
H(\lambda)=\operatorname{det}\left\{\left[\boldsymbol{\Phi}_{\mathbf{u}}(\overline{\mathbf{u}})\right]^{-1}\right\} q\left(\alpha_{21}, \alpha_{32} ; \lambda\right)
$$

and $\operatorname{det}\left\{\left[\boldsymbol{\Phi}_{\mathbf{u}}(\overline{\mathbf{u}})\right]^{-1}\right\}>0$.

For $t=1$, by (4.4), (4.7) and (4.9), we have

$$
\left\{\begin{array}{l}
H\left(\lambda_{0}\right)=H(0)>0, \\
H\left(\lambda_{i}\right)<0 \text { when } 1 \leq i \leq n, \\
H\left(\lambda_{i}\right)>0 \text { when } i>n .
\end{array}\right.
$$

Thus, 0 is not an eigenvalue of the matrix $\lambda_{i} \mathbf{I}-\left[\boldsymbol{\Phi}_{\mathbf{u}}(\overline{\mathbf{u}})\right]^{-1} \mathbf{F}_{\mathbf{u}}(\overline{\mathbf{u}})$ for all $i \geq 0$, and

$$
\sum_{\substack{i \geq 1 \\ H\left(\lambda_{i}\right)<0}} \operatorname{dim} E\left(\lambda_{i}\right)=\sum_{i=1}^{n} \operatorname{dim} E\left(\lambda_{i}\right)=\sigma_{n}
$$

is odd. It follows from Proposition 4.1 that

$$
\operatorname{index}(\Psi(1 ; \cdot), \overline{\mathbf{u}})=(-1)^{r}=(-1)^{\sigma_{n}}=-1 .
$$

For $t=0$, we have $H\left(\lambda_{i}\right)>0$ for all $i \geq 0$. Similarly, we can prove that

$$
\operatorname{index}(\Psi(0 ; \cdot), \overline{\mathbf{u}})=(-1)^{0}=1 \text {. }
$$

On the other hand, by Proposition 3.3 and Proposition 3.4, there exists a positive constant $M$ such that for all $t \in[0,1]$, the positive solution of (4.8) satisfy $M^{-1}<u_{1}, u_{2}, u_{3}<M$ and $\Psi(t ; \mathbf{u}) \neq 0$ on $\partial B(M)$. By the homotopy invariance of the topological degree, we can obtain

$$
\operatorname{deg}(\Psi(1 ; \cdot), 0, B(M))=\operatorname{deg}(\Psi(0 ; \cdot), 0, B(M))
$$

Suppose, on the contrary, that the assertion is not true for some $\alpha_{21}=\bar{\alpha}_{21} \geq \alpha_{21}^{*}$. Let $\alpha_{21}$ be fixed as $\bar{\alpha}_{21}$. Now, by our supposition, 
both equations $\Psi(1 ; \mathbf{u})=0$ and $\Psi(0 ; \mathbf{u})=0$ have only the constant positive solution $\overline{\mathbf{u}}$ in $B(M)$. Thus, by (4.10) and (4.11),

$$
\begin{aligned}
& \operatorname{deg}(\Psi(1 ; \cdot), 0, B(M))=\operatorname{index}(\Psi(1 ; \cdot), \overline{\mathbf{u}})=-1, \\
& \operatorname{deg}(\Psi(0 ; \cdot), 0, B(M))=\operatorname{index}(\Psi(0 ; \cdot), \overline{\mathbf{u}})=1,
\end{aligned}
$$

which contradicts (4.12). The proof is completed.

By a similar approach as in the proof of Theorem 4.4, we can acquire the following result about existence.

Theorem 4.5. Assume that parameters $d_{i}, a_{i}, b_{i i}, i=1,2,3, \alpha_{12}, \alpha_{21}$, $\alpha_{23}, b_{12}, b_{21}$ and $b_{32}$ are all fixed and satisfy (2.2) and (4.5). Let $\bar{\lambda}^{\prime}$ be given by the limit (4.6). If $\bar{\lambda}^{\prime} \in\left(\lambda_{n}, \lambda_{n+1}\right)$ for some $n \geq 1$, and the sum $\sigma_{n}=\sum_{i=1}^{n} \operatorname{dim} E\left(\lambda_{i}\right)$ is odd, then there exists a positive constant $\alpha_{32}^{*}$ such that problem (1.1) has at least one non-constant positive steady state provided that $\alpha_{32}>\alpha_{32}^{*}$ and

$$
a_{3} b_{23}>a_{2} b_{33}\left(1+\frac{\alpha_{32}}{d_{3}} M_{2}\right) C_{3}
$$

hold true.

Remark 4.6. Theorems 4.4 and 4.5 indicate that when inter-specific interaction $b_{23}$ is big enough and cross-diffusion $\alpha_{21}$ or $\alpha_{32}$ is large, it is easy to create non-constant positive steady states (stationary patterns).

Acknowledgments. The authors greatly appreciate the referee for helpful comments and suggestions.

\section{REFERENCES}

1. L. Chen and A. Jüngel, Analysis of a parabolic cross-diffusion population model without self-diffusion, J. Diff. Equat. 224 (2006), 39-59.

2. W. Chen and R. Peng, Stationary patterns created by cross-diffusion for the competitor-competitor-mutualist model, J. Math. Anal. Appl. 291 (2004), 550-564.

3. X. Chen, Y. Qi and M. Wang, A strongly coupled predator-prey system with non-monotonic functional response, Nonl. Anal. TMA 67 (2007), 1966-1979.

4. E.N. Dancer, A counterexample of competing species equations, Diff. Int. Equat. 9 (1996), 239-246. 
5. B. Dubey and B. Hussain, A predator-prey interaction model with self and cross-diffusion, Ecol. Model. 141 (2001), 67-76.

6. S. Fu, Z. Wen and S. Cui, On global solutions for the three-species food-chain model with cross-diffusion, Acta Math. Sinica 50 (2007), 75-88.

7. , Uniform boundedness and stability of global solutions in a strongly coupled three-species cooperating model, Nonl. Anal. RWA 9 (2008), 272-289.

8. G. Galiano, M.L. Garzón and A. Jüngel, Semi-discretization in time and numerical convergence of solutions of a nonlinear cross-diffusion population model, Numer. Math. 93 (2003), 655-673.

9. D. Gilbarg and N.S. Trudinger, Elliptic partial differential equations of second order, Springer, New York, 1977.

10. J.K. Hale, Ordinary differential equations, Krieger, Malabar Florida, 1980.

11. L. Hei, Global bifurcation of co-existence states for a predator-prey-mutualist model with diffusion, Nonl. Anal. RWA 8 (2007), 619-635.

12. T. Kadota and K. Kuto, Positive steady states for a prey-predator model with some nonlinear diffusion terms, J. Math. Anal. Appl. 323 (2006), 1387-1401.

13. Y. Kan-on, Existence and instability of Neumann layer solutions for a 3component Lotka-Volterra model with diffusion, J. Math. Anal. Appl. 243 (2000), $357-372$.

14. Y. Kan-on and M. Mimura, Singular perturbation approach to a 3component reaction-diffusion system arising in population dynamics, SIAM J. Math. Anal. 29 (1998), 1519-1536.

15. K. Ik Kim and Z. Lin, Coexistence of three species in a strongly coupled elliptic system, Nonl. Anal. 55 (2003), 313-333.

16. K. Kuto, Bifurcation branch of stationary solutions for a Lotka-Volterra cross-diffusion system in a spatially heterogeneous environment, Nonl. Anal. RWA 10 (2009), 943-965.

17. K. Kuto and Y. Yamada, Multiple coexistence states for a prey-predator system with cross-diffusion, J. Diff. Equat. 197 (2004), 315-348.

18. D. Le, L.V. Nguyen and T.T. Nguyen, Regularity and coexistence problems for strongly coupled elliptic systems, Ind. Univ. Math. J. 56 (2007), 1749-1791.

19. , Shigesada-Kawasaki-Teramoto model on higher dimensional domains, Electr. J. Diff. Equat. 2003 (2003), 1-12.

20. J. Lopez-Gomez and R. Pardo San Gil, Coexistence in a simple food chain with diffusion, J. Math. Biol. 30 (1992), 655-668.

21. Y. Lou, S. Martínez and W. Ni, On $3 \times 3$ Lotka-Volterra competition systems with cross-diffusion, Discr. Contin. Dyn. Syst. 6 (2000), 175-190.

22. Y. Lou and W. Ni, Diffusion, self-diffusion and cross-diffusion, J. Diff. Equat. 131 (1996), 79-131.

23. 154 (1999), 157-190.

Diffusion vs. cross-diffusion: An elliptic approach, J. Diff. Equat.

24. J.D. Murray, Mathematical biology I: An introduction, third edition, Springer, New York, 2002. 
25. P.Y.H. Pang and M. Wang, Strategy and stationary pattern in a three-species predator-prey model, J. Diff. Equat. 200 (2004), 245-273.

26. C.V. Pao, Strongly coupled elliptic systems and applications to LotkaVolterra models with cross-diffusion, Nonl. Anal. TMA 60 (2005), 1197-1217.

27. R. Peng, M. Wang and M. Yang, Positive solutions of a diffusive preypredator model in a heterogeneous environment, Math. Comp. Model. 46 (2007), 1410-1418.

28. W.H. Ruan, Positive steady-state solutions of a competing reaction-diffusion system with large cross-diffusion coefficients, J. Math. Anal. Appl. 197 (1996), 558578 .

29. N. Shigesada, K. Kawasaki and E. Teramoto, Spatial segregation of interacting species, J. Theor. Biol. 79 (1979), 83-99.

30. A. Turing, The chemical basis of morphogenesis, Philos. Trans. Roy. Soc. 237 (1952), 37-72.

31. $\mathrm{Y}$. Wu, The instability of spiky steady states for a competing species model with cross diffusion, J. Diff. Equat. 213 (2005), 289-340.

32. X. Zeng, Non-constant positive steady states of a prey-predator system with cross-diffusions, J. Math. Anal. Appl. 332 (2007), 989-1009.

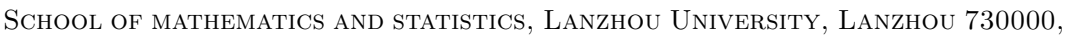
PR CHINA

Email address: wenzj@lzu.edu.cn

Department of Mathematics, Longdong University, Qingyang 745000, PR CHINA

Email address: mathqy@163.com 